International Journal of Pure and Applied Mathematics

Volume 88 No. 3 2013, 425-435

ISSN: 1311-8080 (printed version); ISSN: 1314-3395 (on-line version)

url: http://www.ijpam.eu

doi: http://dx.doi.org/10.12732/ijpam.v88i3.10

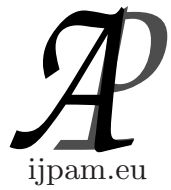

\title{
ON THE CURVATURE FORMS OF NIL SPACE
}

Zühal Küçükarslan Yüzbaş1 ${ }^{1,2} \S$, Mehmet Bektaş², Münevver Yildirim Yilmaz ${ }^{2}$

${ }^{1}$ Department of Mathematics

Brock University

St. Catharines, Ontario, L2S 3A1, CANADA

${ }^{2}$ Department of Mathematics

Faculty of Science

Firat University

23119, Elazig, TURKEY

Abstract: In this work, we study structure equations in Nil space. Also, the submanifold of $N i l$ space is given and sectional, Ricci curvatures in this submanifold are obtained by using Nil metric.

AMS Subject Classification: 53C25, 53C40

Key Words: Nil space, structure equations, submanifold, totally geodesic, sectional and Ricci curvatures

\section{Introduction}

A Riemann manifold is called homogeneous such that for every two point $p$ and $q$ in $M$, there exist an isometry mapping $p$ into $q$. The study of Homogeneous geometries has played a main role in the development modern theory of three manifolds. The famous conjecture of Thurston on the classification of a compact 3 -manifolds in eight 'model geometries', that is, $E^{3}, H^{3}, S^{3}, S^{2} \times R, H^{2} \times$ $R, S L(2, R), N i l$ and $S o l$ in [6].

Received: July 22, 2013

(C) 2013 Academic Publications, Ltd.

$\S$ Correspondence author url: www.acadpubl.eu 
Our aim is just to study the $N i l$ geometry. The left invariant metric on $\mathrm{Nil}_{3}$ defined by

$$
d s^{2}=d x^{2}+d y^{2}+(d z+\tau(y d x-x d y))^{2},
$$

here $\tau$ is non-zero real number. On the other hand biharmonic maps into $N i l$ space is examined in [5], higher order paralel surfaces in Nil space is studied in [7] and different aspect is investigated in [2], [5].

In this paper, first of all the structure equations in Nil space is given, secondly submanifold of Nil space is studied and these manifolds obtain that are not totally geodesic. Finally, sectional and Ricci curvatures of this submanifold are obtained.

\section{Structure Equations of Nil Space}

Let $\left(R^{3}, g_{N i l}\right)$ denote Nil space such that the left-invariant metric can be written as

$$
g_{N i l}=d s^{2}=d x^{2}+d y^{2}+(d z+\tau(y d x-x d y))^{2},
$$

where $\tau$ is non-zero real number. This metric can be written as

$$
d s^{2}=\sum_{1}^{3} w^{i} \otimes w^{i},
$$

where

$$
w^{1}=d x, \quad w^{2}=d y, \quad w^{3}=d z+\tau(y d x-x d y),
$$

and the orthonormal basis of dual vector fields to the 1-form is

$$
\begin{aligned}
& e_{1}=\frac{\partial}{\partial x}-\tau y \frac{\partial}{\partial z}, \\
& e_{2}=\frac{\partial}{\partial y}+\tau x \frac{\partial}{\partial z}, \\
& e_{3}=\frac{\partial}{\partial z} .
\end{aligned}
$$

The corresponding Lie brackets are

$$
\left[e_{1}, e_{2}\right]=2 \tau e_{3}, \quad\left[e_{2}, e_{3}\right]=0, \quad\left[e_{3}, e_{1}\right]=0 .
$$

Levi-civita connection of $\mathrm{Nil}_{3}$ is given by

$$
\begin{array}{ccc}
\tilde{\nabla}_{e_{1}} e_{1}=0, & \tilde{\nabla}_{e_{1}} e_{2}=\tau e_{3}, & \tilde{\nabla}_{e_{1}} e_{3}=-\tau e_{2}, \\
\tilde{\nabla}_{e_{2}} e_{1}=-\tau e_{3}, & \tilde{\nabla}_{e_{2}} e_{2}=0, & \tilde{\nabla}_{e_{2}} e_{3}=\tau e_{1}, \\
\tilde{\nabla}_{e_{3}} e_{1}=-\tau e_{2}, & \tilde{\nabla}_{e_{3}} e_{2}=\tau e_{1}, & \tilde{\nabla}_{e_{3}} e_{3}=0,
\end{array}
$$


in [7]. The non-vanishing Christoffel symbols are obtained by

$$
\Gamma_{12}^{3}=-\Gamma_{21}^{3}=\Gamma_{23}^{1}=\Gamma_{32}^{1}=-\Gamma_{13}^{2}=-\Gamma_{31}^{2}=\tau .
$$

Let $\left\{e_{1}, e_{2}, e_{3}\right\}$ be a local orthonormal frame on $N i l_{3}$ and $\left\{w^{1}, w^{2}, w^{3}\right\}$ be the dual basis $\left\{e_{1}, e_{2}, e_{3}\right\}$, for $1 \leq i, j \leq 3$, the connection form $w_{j}^{i}$ are calculated by

$$
w_{j}^{i}=\sum_{k} g_{N i l} e\left({ }_{i}, \tilde{\nabla}_{e_{\mathrm{k}}} e_{j}\right) w^{k}=\sum_{k} \Gamma_{k j}^{i} w^{k},
$$

in [3]. Then, we obtain the connection form $w_{j}^{i}$ on $\mathrm{Nil}_{3}$ as follows

$$
\begin{array}{ccc}
w_{1}^{1}=0, & w_{1}^{2}=-\tau w^{3}, & w_{1}^{3}=-\tau w^{2}, \\
w_{2}^{1}=\tau w^{3}, & w_{2}^{2}=0, & w_{2}^{3}=\tau w^{1}, \\
w_{3}^{1}=\tau w^{2}, & w_{3}^{2}=-\tau w^{1} & w_{3}^{3}=0 .
\end{array}
$$

The Riemannian curvature tensor $R$ of $\mathrm{Nil}_{3}$ space is defined by using the following convention

$$
\tilde{R}(X, Y) Z=\tilde{\nabla}_{Y} \tilde{\nabla}_{X} Z-\tilde{\nabla}_{X} \tilde{\nabla}_{Y} Z+\tilde{\nabla}_{[X, Y]} Z,
$$

or

$$
\begin{aligned}
\tilde{R}(X, Y) Z= & -3 \tau^{2}\left(g_{N i l}(X, Z) Y-g_{N i l}(Y, Z) X\right) \\
& +4 \tau^{2}\left(g_{N i l}\left(X, e_{3}\right) g_{N i l}\left(Z, e_{3}\right) Y\right. \\
& -g_{N i l}\left(Y, e_{3}\right) g_{N i l}\left(Z, e_{3}\right) X+g_{N i l}\left(Y, e_{3}\right) g_{N i l}(X, Z) e_{3} \\
& \left.-g_{N i l}\left(X, e_{3}\right) g_{N i l}(Y, Z) e_{3}\right),
\end{aligned}
$$

where $X, Y, Z \in T_{p}\left(N i l_{3}\right)$. Then, for $1 \leq i, j, k \leq 3$, we can write the nonvanishing Riemannian curvature tensor $R_{i j k}$ as follow

$$
\begin{array}{cl}
\tilde{R}_{121}=-\tilde{R}_{211}=-3 \tau^{2} e_{1}, & \tilde{R}_{122}=-\tilde{R}_{212}=3 \tau^{2} e_{1}, \\
\tilde{R}_{131}=-\tilde{R}_{311}=\tau^{2} e_{3}, & \tilde{R}_{133}=-\tilde{R}_{313}=-\tau^{2} e_{1}, \\
\tilde{R}_{232}=-\tilde{R}_{321}=\tau^{2} e_{3}, & \tilde{R}_{233}=-\tilde{R}_{323}=-\tau^{2} e_{2} .
\end{array}
$$

Also, for $1 \leq i, j, k, l \leq 3$,

$$
\tilde{R}_{k l j}^{i}=g_{N i l}\left(\tilde{R}\left(e_{k}, e_{l}\right) e_{j}, e_{i}\right) .
$$

Considering equation (2.7), the non-vanishing Riemann curvature tensor $R_{k l j}^{i}$ on $\mathrm{Nil}_{3}$ obtained in the following way

$$
\begin{array}{cc}
\tilde{R}_{121}^{2}=-\tilde{R}_{211}^{2}=-3 \tau^{2}, & \tilde{R}_{122}^{1}=-\tilde{R}_{212}^{1}=3 \tau^{2}, \\
\tilde{R}_{131}^{3}=-\tilde{R}_{311}^{3}=\tau^{2}, & \tilde{R}_{133}^{1}=-\tilde{R}_{313}^{1}=-\tau^{2}, \\
\tilde{R}_{232}^{3}=-\tilde{R}_{321}^{3}=\tau^{2}, & \tilde{R}_{233}^{2}=-\tilde{R}_{323}^{2}=-\tau^{2} .
\end{array}
$$


The structure equations, for $1 \leq i, j, k, l, p \leq 3$,

$$
\begin{aligned}
& d w^{i}=-\sum_{p} w_{p}^{i} \wedge w^{p}, \\
& d w_{j}^{i}=-\sum_{p} w_{p}^{i} \wedge w_{j}^{p}-\frac{1}{2} \sum_{k} \sum_{l} \tilde{R}_{k l j}^{i} w^{k} \wedge w^{l},
\end{aligned}
$$

in [3], [8]. Using above equations, we can show the following;

$$
\begin{aligned}
& d w^{1}=0, \quad d w^{2}=0, \quad d w^{3}=-2 \tau w^{1} \wedge w^{2} . \\
& d w_{1}^{1}=0, \quad d w_{1}^{2}=2 \tau^{2} w^{1} \wedge w^{2}, \quad d w_{1}^{3}=0, \\
& d w_{2}^{1}=-2 \tau^{2} w^{1} \wedge w^{2}, \quad d w_{2}^{2}=0, \quad d w_{2}^{3}=0, \\
& d w_{3}^{1}=0, \quad d w_{3}^{2}=0, \quad d w_{3}^{3}=0 .
\end{aligned}
$$

\section{Sectional and Ricci Curvatures in Submanifold of $\mathrm{Nil}_{3}$ Space}

Let $\left\{e_{1}, e_{2}, e_{3}\right\}$ be a local orthonormal frame on $N i l_{3}$ and $\left\{w^{1}, w^{2}, w^{3}\right\}$ be the dual basis $\left\{e_{1}, e_{2}, e_{3}\right\}$, for $1 \leq i, j \leq 3$. The Riemannian curvature tensor $R$ of $\mathrm{Nil}_{3}$ is given by equation in (2.5).

There are three cases for the submanifold of $\mathrm{Nil}_{3}$ :

Case 1.Let $M_{1}$ be differentiable 2-manifold of $N i l_{3}$ space. Suppose that $e_{3}$ is normal at $M_{1}$, that is, $e_{3} \in \chi\left(M_{1}\right)^{\perp}$. Then

$$
\begin{aligned}
& \tilde{e}_{1}=a_{11} e_{1}+a_{12} e_{2}, \\
& \tilde{e}_{2}=a_{21} e_{1}+a_{22} e_{2},
\end{aligned}
$$

where $\tilde{e}_{1}, \tilde{e}_{2}$ are basis vector of $M_{1}$ and for $1 \leq i, j \leq 2, a_{i j}$ are real valued differentiable functions such that $A=\left[a_{i j}\right]$ and $\operatorname{det} A \neq 0$.

Corollary 3.1. $M_{1}$ can not be totally geodesic submanifold of $\mathrm{Nil}_{3}$.

Proof. Let $\tilde{\nabla}^{\prime}$ and $\tilde{\nabla}$ be connection $M_{1}$ and $N i l_{3}$, respectively, and $\tilde{I I}$ be second fundemental form tensor. Then, Gauss equation can be written as follow

$$
\tilde{\nabla}_{\tilde{e}_{\mathrm{i}}} \tilde{e}_{j}=\tilde{\nabla}_{\tilde{e}_{\mathrm{i}}}^{\prime} \tilde{e}_{j}+\tilde{I I}\left(\tilde{e}_{i}, \tilde{e}_{j}\right), \quad 1 \leq i, j \leq 2,
$$

and so obtained

$$
\tilde{I I}\left(\tilde{e}_{i}, e_{j}\right)=\operatorname{nor} \tilde{\nabla}_{\tilde{e}_{i}} \tilde{e}_{j} .
$$


Hence

$$
\begin{gathered}
\tilde{I I}\left(\tilde{e}_{1}, \tilde{e}_{1}\right)=\operatorname{nor} \tilde{\nabla}_{\tilde{e}_{1}} \tilde{e}_{1}=0 . \\
\tilde{I I}\left(\tilde{e}_{1}, \tilde{e}_{2}\right)=\operatorname{nor} \tilde{\nabla}_{\tilde{e}_{1}} \tilde{e}_{2}=\operatorname{det} A \tau e_{3} . \\
\tilde{I I}\left(\tilde{e}_{2}, \tilde{e}_{1}\right)=\operatorname{nor} \tilde{\nabla}_{\tilde{e}_{2}} \tilde{e}_{1}=-\operatorname{det} A \tau e_{3} . \\
\tilde{I I}\left(\tilde{e}_{2}, \tilde{e}_{2}\right)=\operatorname{nor} \tilde{\nabla}_{\tilde{e}_{2}} \tilde{e}_{2}=0 .
\end{gathered}
$$

If $M_{1}$ is totally geodesic, $\tilde{I I}$ is zero and $\tau \neq 0 . \tilde{I I}$ equals to zero in $(3.2),(3.3),(3.4)$ and $(3.5)$, we get

$$
a_{21} a_{12}-a_{22} a_{11}=0
$$

this means that $\operatorname{det} A=0$. This is a contradiction, that is, $M_{1} \subset N i l_{3}$ can not be totally geodesic.

Corollary 3.2. The sectional curvature $\tilde{K}_{1}$ of $M_{1} \subset N i l_{3}$ is given by

$$
\tilde{K}_{1}=\frac{a_{21}\left(B_{1}-D_{1}+F_{1}\right)+a_{22}\left(B_{2}-D_{2}+F_{2}\right)}{(\operatorname{det} A)^{2}},
$$

where

$B_{1}=a_{21} e_{1}\left[A_{1}\right]+a_{22} e_{2}\left[A_{1}\right], B_{2}=a_{21} e_{1}\left[A_{2}\right]+a_{22} e_{2}\left[A_{2}\right], B_{3}=\left(a_{21} A_{2}-a_{22} A_{1}\right) \tau$ $\left(A_{1}=a_{11} e_{1}\left[a_{11}\right]+a_{12} e_{2}\left[a_{11}\right], A_{2}=a_{11} e_{1}\left[a_{12}\right]+a_{12} e_{2}\left[a_{12}\right], A_{3}=0\right)$, $D_{1}=a_{11} e_{1}\left[C_{1}\right]+a_{12} e_{2}\left[C_{1}\right]+a_{12} C_{3} \tau, D_{2}=a_{11} e_{1}\left[C_{2}\right]+a_{12} e_{2}\left[C_{2}\right]-a_{11} C_{3} \tau$, $D_{3}=a_{11} e_{1}\left[C_{3}\right]+a_{12} e_{2}\left[C_{3}\right]-a_{12} C_{1} \tau+a_{11} C_{2} \tau$, $\left(C_{1}=a_{21} e_{1}\left[a_{11}\right]+a_{22} e_{2}\left[a_{11}\right], C_{2}=a_{21} e_{1}\left[a_{12}\right]+a_{22} e_{2}\left[a_{12}\right], C_{3}=-\tau \operatorname{det} A.\right)$, $F_{1}=2 \tau \operatorname{det} A\left(e_{3}\left[a_{11}\right]+\tau a_{12}\right), \quad F_{2}=2 \tau \operatorname{det} A\left(e_{3}\left[a_{12}\right]-\tau a_{11}\right)$.

Proof. We know that

$$
\tilde{K}_{1}\left(\tilde{e}_{1}, \tilde{e}_{2}\right)=\frac{g_{N i l}\left(\tilde{R}\left(\tilde{e}_{1}, \tilde{e}_{2}\right) \tilde{e}_{1}, \tilde{e}_{2}\right)}{g_{N i l}\left(\tilde{e}_{1}, \tilde{e}_{1}\right) g_{N i l}\left(\tilde{e}_{2}, \tilde{e}_{2}\right)-g_{N i l}\left(\tilde{e}_{1}, \tilde{e}_{2}\right)^{2}},
$$

hence, by a straightforward computation, we have

$$
\tilde{K}_{1}=\frac{a_{21}\left(B_{1}-D_{1}+F_{1}\right)+a_{22}\left(B_{2}-D_{2}+F_{2}\right)}{(\operatorname{det} A)^{2}} .
$$

Corollary 3.3. For $1 \leq i, j, k \leq 3$, if $e_{k}\left[a_{i j}\right]$ is zero, then $\tilde{K}_{1}=-3 \tau^{2}$, where $e_{k}\left[a_{i j}\right]$ is the directional derivatives of the functions $a_{i j}$ in terms of the vector $e_{k}$. 
Proof. Since $e_{k}\left[a_{i j}\right]=0$, from Corollary 3.2, we get

$$
\begin{aligned}
B_{1} & =0, B_{2}=0, \\
D_{1} & =-\tau^{2} a_{12} \operatorname{det} A, D_{2}=\tau^{2} a_{11} \operatorname{det} A, \\
F_{1} & =2 \tau^{2} a_{12} \operatorname{det} A, F_{2}=-2 \tau^{2} a_{11} \operatorname{det} A .
\end{aligned}
$$

we substitue (3.7) into (3.6), we have $\tilde{K}_{1}=-3 \tau^{2}$.

By Gauss Bonnet theorem, we give the folllowing result.

Corollary 3.4. If $M_{1}$ is compact, then the Euler characteristic of $M_{1}$ is negative.

Corollarry 3.5. The Ricci curvature of $M_{1}$ is always negative.

Proof. Let $p \in M_{1}, \tilde{e}_{1}, \tilde{e}_{2} \in T_{p}\left(M_{1}\right)$ and $e_{3} \in T_{p}\left(M_{1}\right)^{\perp}$,

$$
\operatorname{Ric}_{1}\left(e_{3}\right)=\frac{1}{2} \sum_{i}\left\langle\tilde{R}\left(e_{3}, \tilde{e}_{i}\right) e_{3}, \tilde{e}_{i}\right\rangle=-\frac{\tau^{2}}{2} \sum_{i, j} a_{i j}^{2}, \quad 1 \leq i, j \leq 2,
$$

Furthermore computation leads to

$$
\operatorname{Ric}_{1}\left(e_{3}\right)=-\frac{\tau^{2}}{2} \sum_{i, j} a_{i j}^{2}, \quad 1 \leq i, j \leq 2 .
$$

Case 2. Let $M_{2}$ be differentiable 2-manifold of $N i l_{3}$ space. Suppose that $e_{2}$ is normal at $M_{2}$, that is, $e_{2} \in \chi\left(M_{2}\right)^{\perp}$. Then

$$
\begin{aligned}
& \tilde{e}_{1}=b_{11} e_{1}+b_{12} e_{3}, \\
& \tilde{e}_{2}=b_{21} e_{1}+b_{22} e_{3},
\end{aligned}
$$

where $\tilde{e}_{1}, \tilde{e}_{2}$ are basis vector of $M_{2}$ and for $1 \leq i, j \leq 2, b_{i j}$ are real valued differentiable functions such that $B=\left[b_{i j}\right]$ and $\operatorname{det} B \neq 0$.

Corollary 3.6. $M_{2}$ can not be totally geodesic submanifold of $\mathrm{Nil}_{3}$.

Proof. Let $\tilde{\nabla}^{\prime}$ and $\tilde{\nabla}$ be connection $M_{2}$ and $N i l_{3}$, respectively, and $\tilde{I I}$ be second fundemental form tensor. Then, from (3.1),

$$
\begin{gathered}
\tilde{I I}\left(\tilde{e}_{1}, \tilde{e}_{1}\right)=\operatorname{nor} \tilde{\nabla}_{\tilde{e}_{1}} \tilde{e}_{1}=-\left(2 b_{11} b_{12}\right) \tau e_{2} \\
\tilde{I I}\left(\tilde{e}_{1}, \tilde{e}_{2}\right)=\operatorname{nor} \tilde{\nabla}_{\tilde{e}_{1}} \tilde{e}_{2}=\left(b_{11} b_{22}+b_{12} b_{21}\right) \tau e_{2} .
\end{gathered}
$$




$$
\begin{gathered}
\tilde{I I}\left(\tilde{e}_{2}, \tilde{e}_{1}\right)=\operatorname{nor} \tilde{\nabla}_{\tilde{e}_{2}} \tilde{e}_{1}=\left(b_{21} b_{12}+b_{22} b_{11}\right) \tau e_{2} . \\
\tilde{I I}\left(\tilde{e}_{2}, \tilde{e}_{2}\right)=\operatorname{nor} \tilde{\nabla}_{\tilde{e}_{2}} \tilde{e}_{2}=-\left(2 b_{21} b_{22}\right) \tau e_{2} .
\end{gathered}
$$

If $M_{2}$ is totally geodesic, $\tilde{I I}$ is zero and $\tau \neq 0$. $\tilde{I I}$ equals to zero in $(3.9),(3.10),(3.11)$ and (3.12), we get

$$
\begin{aligned}
b_{21} b_{12}+b_{22} b_{11} & =0, \\
b_{11} b_{12} & =0, \\
b_{21} b_{22} & =0,
\end{aligned}
$$

which means $\operatorname{det} B=0$. This is a contradiction, so $M_{2} \subset N i l_{3}$ can not be totally geodesic.

Corollary 3.7. The sectional curvature $\tilde{K}_{2}$ of $M_{2} \subset N i l_{3}$ is given by

$$
\tilde{K}_{2}=\frac{b_{21}\left(H_{1}-J_{1}\right)+b_{22}\left(H_{3}-J_{3}\right)}{(\operatorname{det} B)^{2}},
$$

where $H_{1}=b_{21} e_{1}\left[G_{1}\right]+b_{22} e_{3}\left[G_{1}\right]+b_{22} G_{2} \tau$,

$H_{2}=b_{21} e_{1}\left[G_{2}\right]+b_{22} e_{3}\left[G_{2}\right]-b_{21} G_{3} \tau-b_{22} G_{1} \tau$,

$H_{3}=b_{21} e_{1}\left[G_{3}\right]+b_{22} e_{3}\left[G_{3}\right]+b_{21} G_{2} \tau$,

$\left(G_{1}=b_{11} e_{1}\left[b_{11}\right]+b_{12} e_{3}\left[b_{11}\right], G_{2}=-2 \tau b_{11} b_{12}, G_{3}=b_{11} e_{1}\left[b_{12}\right]+b_{12} e_{3}\left[b_{12}\right]\right)$,

$J_{1}=b_{11} e_{1}\left[I_{1}\right]+b_{12} e_{3}\left[I_{1}\right]+b_{12} I_{2} \tau$,

$J_{2}=b_{11} e_{1}\left[I_{2}\right]+b_{12} e_{3}\left[I_{2}\right]-b_{12} I_{1} \tau-b_{11} I_{3} \tau$,

$J_{3}=b_{11} e_{1}\left[I_{3}\right]+b_{12} e_{3}\left[I_{3}\right]+b_{11} I_{2} \tau$,

$\left(I_{1}=b_{21} e_{1}\left[b_{11}\right]+b_{22} e_{3}\left[b_{11}\right], I_{2}=-\left(b_{21} b_{12}+b_{22} b_{11}\right) \tau, I_{3}=b_{21} e_{1}\left[b_{12}\right]+a_{22} e_{3}\left[b_{12}\right]\right)$.

Proof. We know that

$$
\tilde{K}_{2}\left(\tilde{e}_{1}, \tilde{e}_{2}\right)=\frac{g_{N i l}\left(\tilde{R}\left(\tilde{e}_{1}, \tilde{e}_{2}\right) \tilde{e}_{1}, \tilde{e}_{2}\right)}{g_{N i l}\left(\tilde{e}_{1}, \tilde{e}_{1}\right) g_{N i l}\left(\tilde{e}_{2}, \tilde{e}_{2}\right)-g_{N i l}\left(\tilde{e}_{1}, \tilde{e}_{2}\right)^{2}},
$$

and so this proof can be easily shown by using above equation.

Corollary 3.8. For $1 \leq i, j, k \leq 3$, if $e_{k}\left[a_{i j}\right]$ is zero, then $\tilde{K}_{2}=\tau^{2}$, where $e_{k}\left[b_{i j}\right]$ is the directional derivatives of the functions $b_{i j}$ in terms of the vector $e_{k}$. 
Proof. Since $e_{k}\left[b_{i j}\right]=0$, from Corollary 3.7, we get

$$
\begin{aligned}
H_{1} & =-2 \tau^{2} b_{11} b_{12} b_{22}, H_{3}=-2 \tau^{2} b_{21} b_{11} b_{12}, \\
J_{1} & =-\tau^{2} b_{12}\left(b_{21} b_{12}+b_{22} b_{11}\right), J_{3}=-\tau^{2} b_{11}\left(b_{21} b_{12}+b_{22} b_{11}\right) .
\end{aligned}
$$

we substitue (3.14) in (3.13), we have $\tilde{K}_{2}=\tau^{2}$.

By Gauss Bonnet theorem, we give the folllowing result.

Corollary 3.9. If $M_{2}$ is compact, then the Euler characteristic of $M_{2}$ is positive.

Corollarry 3.10. The Ricci curvature of $M_{2}$ is given by

$$
\operatorname{Ric}_{2}\left(e_{2}\right)=\frac{\tau^{2}}{2}\left(3 b_{11}^{2}-b_{12}^{2}+3 b_{21}^{2}-b_{22}^{2}\right) \text {. }
$$

Proof. Let $p \in M_{2}, \tilde{e}_{1}, \tilde{e}_{2} \in T_{p}\left(M_{2}\right)$ and $e_{2} \in T_{p}\left(M_{2}\right)^{\perp}$,

$$
\operatorname{Ric}_{2}\left(e_{2}\right)=\frac{1}{2} \sum_{i}\left\langle\tilde{R}\left(e_{2}, \tilde{e}_{i}\right) e_{2}, \tilde{e}_{i}\right\rangle,
$$

Furthermore computation leads to

$$
\operatorname{Ric}_{2}\left(e_{2}\right)=\frac{\tau^{2}}{2}\left(3 b_{11}^{2}-b_{12}^{2}+3 b_{21}^{2}-b_{22}^{2}\right) .
$$

Case 3. Let $M_{3}$ be differentiable 2-manifold of $N i l_{3}$ space. Suppose that $e_{1}$ is normal at $M_{3}$, that is, $e_{1} \in \chi\left(M_{3}\right)^{\perp}$. Then

$$
\begin{aligned}
& \tilde{e}_{1}=c_{11} e_{2}+c_{12} e_{3}, \\
& \tilde{e}_{2}=c_{21} e_{3}+c_{22} e_{3},
\end{aligned}
$$

where $\tilde{e}_{1}, \tilde{e}_{2}$ are basis vector of $M_{3}$ and for $1 \leq i, j \leq 2, c_{i j}$ are real valued differentiable functions such that $C=\left[c_{i j}\right]$ and $\operatorname{det} C \neq 0$.

Corollary 3.11. $M_{3}$ can not be totally geodesic submanifold of $\mathrm{Nil}_{3}$.

Proof. Let $\tilde{\nabla}^{\prime}$ and $\tilde{\nabla}$ be connection $M_{3}$ and $N i l_{3}$, respectively, and $\tilde{I I}$ be second fundemental form tensor. Then, from (3.1),

$$
\tilde{I I}\left(\tilde{e}_{1}, \tilde{e}_{1}\right)=\operatorname{nor} \tilde{\nabla}_{\tilde{e}_{1}} \tilde{e}_{1}=2 b_{11} b_{12} \tau e_{1}
$$




$$
\begin{gathered}
\tilde{I I}\left(\tilde{e}_{1}, \tilde{e}_{2}\right)=\operatorname{nor} \tilde{\nabla}_{\tilde{e}_{1}} \tilde{e}_{2}=\left(c_{11} c_{22}+c_{12} c_{21}\right) \tau e_{1} . \\
\tilde{I I}\left(\tilde{e}_{2}, \tilde{e}_{1}\right)=\operatorname{nor} \tilde{\nabla}_{\tilde{e}_{2}} \tilde{e}_{1}=\left(c_{21} c_{12}+c_{22} c_{11}\right) \tau e_{1} . \\
\tilde{I I}\left(\tilde{e}_{2}, \tilde{e}_{2}\right)=\operatorname{nor} \tilde{\nabla}_{\tilde{e}_{2}} \tilde{e}_{2}=2 b_{21} b_{22} \tau e_{1} .
\end{gathered}
$$

If $M_{3}$ is totally geodesic, $\tilde{I I}$ is zero and $\tau \neq 0 . \tilde{I I}$ equals to zero in $(3.7),(3.8),(3.9)$ and $(3.10)$, we get

$$
\begin{aligned}
c_{21} c_{12}+c_{22} c_{11} & =0, \\
c_{11} c_{12} & =0, \\
c_{21} c_{22} & =0,
\end{aligned}
$$

which means $\operatorname{det} C=0$. This is a contradiction, that is, $M_{3} \subset N i l_{3}$ cannot be totally geodesic.

Corollary 3.12. The sectional curvature $\tilde{K}_{3}$ of $M_{3} \subset N_{i l}$ is

$$
\tilde{K}_{3}=\frac{c_{21}\left(L_{2}-N_{2}\right)+c_{22}\left(L_{3}-N_{3}\right)}{(\operatorname{det} C)^{2}},
$$

where $L_{1}=c_{21} e_{2}\left[K_{1}\right]+c_{22} e_{3}\left[K_{1}\right]+c_{21} K_{3} \tau+c_{22} K_{2} \tau, L_{2}=c_{21} e_{2}\left[K_{2}\right]+c_{22} e_{3}\left[K_{2}\right]-$ $c_{22} M_{1} \tau, L_{3}=c_{21} e_{2}\left[K_{3}\right]+c_{22} e_{3}\left[K_{3}\right]-c_{21} K_{1} \tau,\left(K_{1}=2 \tau c_{11} c_{12}, K_{2}=c_{11} e_{2}\left[c_{11}\right]+\right.$ $\left.c_{12} e_{3}\left[c_{11}\right], K_{3}=c_{11} e_{2}\left[c_{12}\right]+c_{12} e_{3}\left[c_{12}\right]\right), N_{1}=c_{11} e_{2}\left[M_{1}\right]+c_{12} e_{3}\left[M_{1}\right]+c_{11} M_{3} \tau+$ $c_{12} M_{2} \tau, N_{2}=c_{11} e_{2}\left[M_{2}\right]+c_{12} e_{3}\left[M_{2}\right]-c_{12} M_{1} \tau, N_{3}=c_{11} e_{2}\left[M_{3}\right]+c_{12} e_{3}\left[M_{3}\right]-$ $c_{11} M_{1} \tau,\left(M_{1}=\left(c_{21} c_{12}+c_{22} c_{11}\right) \tau, M_{2}=c_{21} e_{2}\left[c_{11}\right]+c_{22} e_{3}\left[c_{11}\right], M_{3}=c_{21} e_{2}\left[c_{12}\right]+\right.$ $\left.c_{22} e_{3}\left[c_{12}\right]\right)$.

Proof. We know that

$$
\tilde{K}_{3}\left(\tilde{e}_{1}, \tilde{e}_{2}\right)=\frac{g_{N i l}\left(\tilde{R}\left(\tilde{e}_{1}, \tilde{e}_{2}\right) \tilde{e}_{1}, \tilde{e}_{2}\right)}{g_{N i l}\left(\tilde{e}_{1}, \tilde{e}_{1}\right) g_{N i l}\left(\tilde{e}_{2}, \tilde{e}_{2}\right)-g_{N i l}\left(\tilde{e}_{1}, \tilde{e}_{2}\right)^{2}},
$$

so this proof can be easily shown by using basic calculation.

Corollary 3.13. For $1 \leq i, j, k \leq 3$, if $e_{k}\left[c_{i j}\right]$ is zero, then $\tilde{K}_{3}=\tau^{2}$, where $e_{k}\left[c_{i j}\right]$ is the directional derivatives of the functions $c_{i j}$ in terms of the vector $e_{k}$.

Proof. Since $e_{k}\left[c_{i j}\right]=0$, from Corollary 3.12, we obtain

$$
L_{2}=-2 \tau^{2} c_{11} c_{12} c_{22}, L_{3}=-2 \tau^{2} c_{21} c_{11} c_{12},
$$




$$
N_{2}=-\tau^{2} c_{12}\left(c_{21} c_{12}+c_{22} c_{11}\right), N_{3}=-\tau^{2} c_{11}\left(c_{21} c_{12}+c_{22} c_{11}\right) .
$$

we substitue $(3.21)$ in $(3.20)$, we have $\tilde{K}_{3}=\tau^{2}$.

By Gauss Bonnet theorem, we have the following result.

Corollary 3.14. If $M_{3}$ is compact, then the Euler characteristic of $M_{3}$ is positive.

Corollarry 3.15. The Ricci curvature of $M_{3}$ is given by

$$
\operatorname{Ric}_{3}\left(e_{1}\right)=\frac{\tau^{2}}{2}\left(3 c_{11}^{2}-c_{12}^{2}+3 c_{21}^{2}-c_{22}^{2}\right) \text {. }
$$

Proof. Let $p \in M_{3}, \tilde{e}_{1}, \tilde{e}_{2} \in T_{p}\left(M_{3}\right)$ and $e_{1} \in T_{p}\left(M_{3}\right)^{\perp}$,

$$
\operatorname{Ric}_{3}\left(e_{1}\right)=\frac{1}{2} \sum_{i}\left\langle\tilde{R}\left(e_{1}, \tilde{e}_{i}\right) e_{1}, \tilde{e}_{i}\right\rangle,
$$

Furthermore computation leads to

$$
\operatorname{Ric}_{3}\left(e_{1}\right)=\frac{\tau^{2}}{2}\left(3 c_{11}^{2}-c_{12}^{2}+3 c_{21}^{2}-c_{22}^{2}\right)
$$

\section{References}

[1] A. Bölcskei, S. Brigitta, Frenet formulas and geodesics in sol geometry, Contributions to Algebra and Geometry, 48 , No. 2 (2007), 411-421.

[2] R. Caddeo, S. Montaldo, C. Oniciuc, P. Piu, The classification of biharmonic curves of Cartan-Vranceanu 3-dimensional spaces, ArXiv:math/0510435v1 (2005).

[3] B. Daniel, Isometric immersion into 3-dimensional homogeneous manifolds, ArXiv:math/0503500v1 (2005).

[4] P.M. Do Carmo, Riemann Geometry, Birkhäuser Boston (1992).

[5] Y. Ou, Z. Wang, Biharmonic maps into Sol and Nil spaces, ArXiv:math/0612329v1 (2006).

[6] W.P. Thurston, Three dimensional manifolds, Kleinian groups and hyperbolic geometry, Bull. Am. Math. Soc., New Ser., 6 (1982), 357-379. 
[7] J. Van der Veken, Higher order parallel surfaces in Bianchi-CartanVranceanu spaces, Result. Math., 51 (2008), 339-359.

[8] K. Yano, M. Kon, Structures on Manifolds, World Scientic Publishing Co. Pte. Ltd. (1984). 
